\title{
Cortando bolo, pizza e grissini
}

\author{
José Antonio Salvador
}

\begin{abstract}
Resumo
Nos processos que levam à aprendizagem matemática destacamos o Ensino da Matemática através da Resolução de Problemas que permitam descobrir padrões, fazer conjecturas e demonstrações explorando diferentes conteúdos. Nesse trabalho investigamos a resolução do problema sobre quantos pedaços de um bolo obtemos com $n=0,1,2, \ldots$ cortes planos e problemas relacionados. A riqueza deste problema permite a exploração de diversos conteúdos matemáticos como geometria, sequências, tabelas, gráficos, primeiras e segundas diferenças, padrões, indução matemática, equações discretas, contagem, arranjos e combinações, grafos e obtenção de coeficientes de polinômios usando resolução de sistemas lineares. Tal abordagem pode contribuir para rever, adquirir e ampliar o conhecimento dos estudantes de uma disciplina do curso de Licenciatura em Matemática ou mesmo do Ensino Médio.
\end{abstract}

Palavras-chave: Resolução de problemas matemáticos; Cortes planos de um bolo; Padrões; Sequências.

\section{Abstract}

In the processes that lead to mathematical learning we highlight the Teaching of Mathematics through Problem Solving which allows us to discover patterns, make conjectures and demonstrate, by exploring different contents. In this paper we investigate the problem of how many pieces of cake we get to make with $n=0,1,2, \ldots$ flat cuts. The richness of this problem allows to explore the various contents such as geometry, sequences, tables, graphs, first and second differences, pattern, mathematical induction, discrete equations, counting, arrangements and combinations, graphs and obtaining polynomial coefficients using resolution of linear systems. This approach can help to revise, acquire and undergrad students' knowledge of a subject in mathematics or even high school students' .

Keywords: Linear cuts of a cake; Mathematical problem solving; Mathematical patterns; Sequences.

\section{Introdução}

Num primeiro momento podemos apresentar a figura de um bolo aos estudantes de uma aula de matemática para despertar a curiosidade. Que perguntas eles fariam? Poderiam imaginar questões sobre vários conteúdos matemáticos, desde o tamanho ou peso do bolo, porcentagens dos ingredientes, nutrientes, calorias, tempo de preparo, custo, divisão, cortes em um certo número de pedaços etc. 
Neste trabalho exploramos o problema de determinar quantos pedaços de bolo podemos obter com $n=0,1,2, \ldots$ cortes planos, que é equivalente a quantas regiões $n$ planos divide o espaço.

Ao orientarmos pequenos grupos de licenciandos ou estudantes do Ensino Médio conduzimos ao entendimento do problema, facilitando com questionamentos para que eles agilizem os seus conhecimentos prévios e que possam descobrir novos.

A princípio, observamos que a maioria deles entende o problema, mas apresenta dificuldades para resolver, exceto quando tentam visualizar e desenhar os cortes em posições particulares, como paralelos ou tendo uma mesma interseção.

No processo de discussão podemos revisar os conceitos relacionados, como plano, espaço, posições relativas de como os planos estão dispostos etc. Questionando sobre o que queremos obter: o número mínimo ou máximo de partes, quais são as possibilidades e que estratégias utilizar. Tentativas e erros, adivinhação, análise geométrica, testando casos iniciais para $n=0,1,2$ cortes no bolo esboçando figuras são as ideias iniciais que geralmente aparecem. Também se podem organizar os dados iniciais obtidos numa tabela e analisá-la, fazer gráficos, procurar padrões, estabelecer uma relação de recorrência e obter uma função discreta, resolvê-la com as condições iniciais. A dificuldade de solucionar o problema para a maioria leva-nos a sugerir uma estratégia de resolver um problema análogo, entendendo primeiro um caso mais simples que pode ser útil na busca da solução, conforme [6] e outros pesquisadores da arte de resolver problemas sugerem.

Um problema investigativo mais simples pode ser o de encontrar o (maior) número de partes em que $n, n \in \mathbb{N}$ (conjunto dos números naturais) cortes retilíneos divide uma pizza grande e fina, conhecido como Pizza de Steiner (equivalente ao problema de quantas regiões $n$ retas dividem um plano) mais fácil de visualizar e de fazer tentativas, experimentos, conjecturas, erros e acertos.

Mais elementar ainda, o problema equivalente em uma dimensão é sobre quantas partes obtém-se com $n$ quebras de um grissini (palito fininho e comprido de pão), ou equivalente, em quantas regiões $n$ pontos divide um segmento de reta (ou uma reta).

Como sabemos, a ênfase da metodologia de resolução de problemas desde as ideias iniciais de compreensão dos mesmos deve ser dada ao processo de resolução, à checagem de cada passo da solução a princípio provisória, e testá-la dispondo-se a submeter os resultados ou conclusões a uma revisão minuciosa.

Descobrindo uma fórmula de recorrência para os problemas em uma dimensão (grissini), duas dimensões (pizza grande e fina) ou três dimensões (bolo), podemos questionar um problema mais geral sobre qual é o maior número de partes que $n$ objetos $k$-dimensionais, $k \in \mathbb{N}$, corta um objeto de uma dimensão superior $(k+1)$ - dimensional.

Nesse sentido, visamos a construção de conceitos e resultados matemáticos através de situações estimuladoras e desafiadoras que percorremos desde a abordagem preliminar do problema: o entendimento do enunciado; a interpretação matemática com linguagem adequada; compreensão dos dados do problema; o que está sendo solicitado; quais as habilidades necessárias para busca da resolução; que conhecimentos prévios poderão ser mobilizados para a resolução do mesmo - se é que ele tem solução; ou a partir de sua resolução? etc. Será que a partir do resultado obtido podemos checar ou demonstrar a solução encontrada, etc.

Poderíamos ainda expressar nosso sentimento indicando se achamos fácil ou difícil, se gostamos ou não, e também dos conceitos e resultados revisitados ou adquiridos com a resolução do problema. 
Abordaremos aqui desde o problema da quebra de um grissini, dos cortes lineares de uma plana fina e finalmente o de cortes planos de um bolo.

\section{Quebrando um grissini $n$ vezes}

Consideramos inicialmente o problema mais simples que consiste em obter o maior número de pedaços ao quebrar um grissini fino e comprido $n$ vezes, $n \in \mathbb{N}$.

Para descobrir o padrão com $n$ quebras de um grissini propomos considerar a relação que associa a cada número natural $n$ de pontos sobre um segmento de reta ou sobre uma reta, o número de pedaços ou regiões $r(n)$ obtidas.

De fato, pode-se testar inicialmente para $n=0$ (zero quebra do grissini ) e obter $r(0)=1$ (parte), ou seja, deixa uma única região linear. Se tivermos $n=1$ quebra, obtemos $r(1)=r(0)+1=$ $1+1=2$, ou seja, uma quebra divide-o em duas partes. Ou seja, um ponto sobre uma linha reta divide-a em duas regiões.

Com $n=2$ (duas quebras), em quaisquer posições do grissini, obtemos 3 partes, ou seja, $r(2)=$ $r(1)+1=2+1=3$.

Podemos construir uma tabela com os valores iniciais para cada passo, conforme Tabela 1, de modo que facilita a descoberta de um padrão;

\begin{tabular}{|c|c|c|c|c|c|c|c|c|c|c|c|}
\hline$n$ & 0 & 1 & 2 & 3 & 4 & 5 & 6 & 7 & $\cdots$ & $\mathrm{n}-1$ & $\mathrm{n}$ \\
\hline \hline$r(n)$ & 1 & 2 & 3 & 4 & 5 & 6 & 7 & 8 & $\cdots$ & $n$ & $\mathrm{n}+1$ \\
\hline$\Delta r(n)$ & 1 & 1 & 1 & 1 & 1 & 1 & 1 & $\cdots$ & 1 & 1 & \\
\hline
\end{tabular}

Tabela 1: $n$ quebras de um grissini, $r(n)$ (partes) e $\Delta r(n)$ (diferença)

$$
r(n)=r(n-1)+1, n \in \mathbb{N} .
$$

E fazer a observação de que a diferença primeira

$$
\Delta r(n)=r(n)-r(n-1), \quad n=1,2,3, \ldots
$$

dos valores de $r(n)$ é constante igual a 1, e, portanto, a sequência $r(n)$ é uma PA (progressão aritmética) com primeiro termo $r(0)=1$ e razão igual a 1 , conforme sugere a Tabela 1 .

A cada quebra de um grissini (não importando o local) obtemos sempre uma parte a mais representada pela função discreta linear $r(n)=1+n$, com $n \in \mathbb{N}$, cujas propriedades e o gráfico afim discreto podem ser explorados como mostra a Figura 1. 


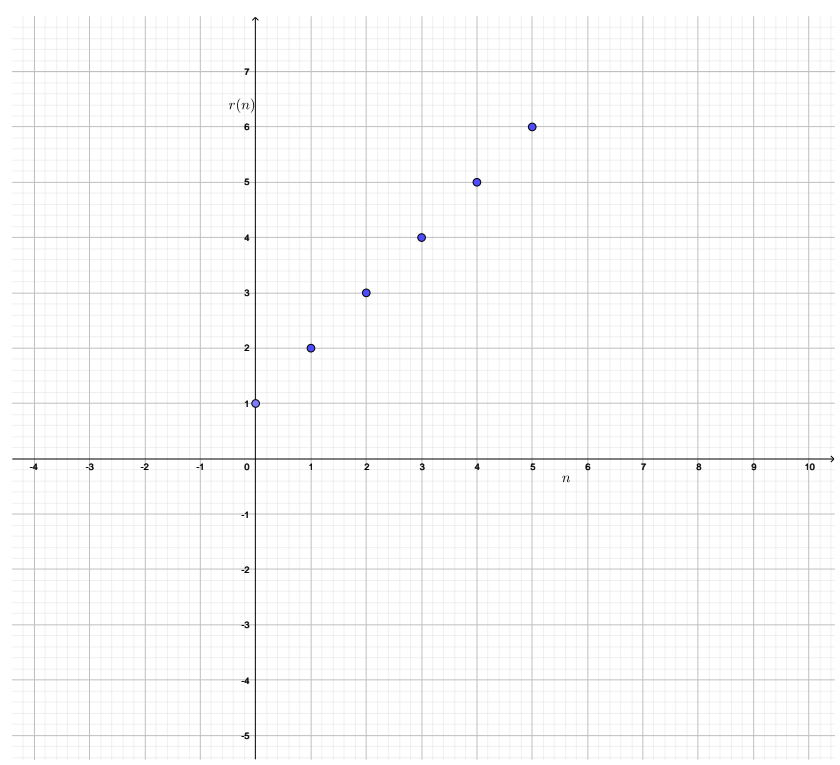

Figura 1: Número de partes de um grissini cortado em $n$ pontos

Podemos provar diretamente, calculando a solução da equação discreta linear de primeira ordem não homogênea $r(n+1)=r(n)+1$ com a condição inicial $r(0)=1$, para obter

$$
r(n)=1+n, \forall n \in \mathbb{N}
$$

partes de um grissini quebrando em $n$ pontos.

Certamente podemos provar de outras maneiras, como pelo Método da Indução Matemática ou, mesmo, contando o número máximo de pedaços obtidos usando combinação,

$$
r(n)=\left(\begin{array}{l}
n \\
0
\end{array}\right)+\left(\begin{array}{l}
n \\
1
\end{array}\right)=1+n
$$

em que $\left(\begin{array}{c}n \\ i\end{array}\right)$ é o número binomial de ordem $n$ e classe $i, i=0,1,2, \ldots, n$, ou seja, o número de subconjuntos distintos com $i$ elementos tomados de um conjunto com $n$ elementos.

\section{Cortes retilíneos de uma pizza}

Entendido o caso dos grissinis, pensamos em quantas partes obtemos com $n$ cortes retilíneos numa enorme pizza considerada plana. Devemos agora considerar vários casos, dependendo das posições relativas dos cortes retilíneos paralelos, concorrentes num mesmo ponto, alguns paralelos e outros concorrentes ou no caso mais geral, cortes concorrentes não se cruzando mais do que dois deles num mesmo ponto conforme Figura 2. 


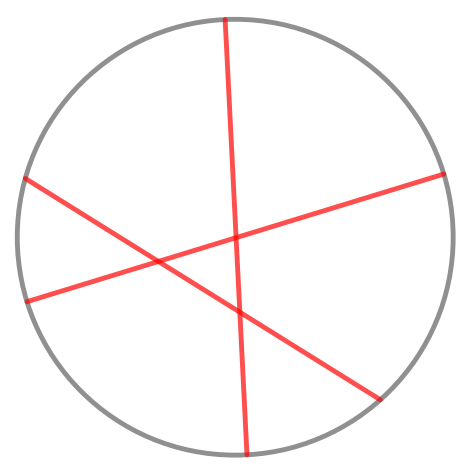

Figura 2: Pizza cortada retilineamente três vezes

\subsection{Cortes paralelos}

Cortes paralelos numa pizza é um problema análogo ao caso das quebras de um grissini; para $n \in \mathbb{N}$ cortes paralelos temos que $p_{p}(n)$ é o número de partes (regiões) que a pizza fica dividida

$$
p_{p}(n)=n+1, \forall n \in \mathbb{N} .
$$

\subsection{Cortes concorrentes num mesmo ponto}

Se todos os $n$ cortes retilíneos distintos forem concorrentes no mesmo ponto central, como geralmente se corta uma pizza circular, podemos elaborar tabela e gráficos $\operatorname{com} n=0,1,2, \ldots$ cortes retilíneos versus $p_{c m p}(n)$, representando o número de regiões que os $n$ cortes concorrentes no mesmo ponto gera,

$$
p_{c m p}(n)=2 n, n \in \mathbb{N},
$$

conforme a Figura 3. 


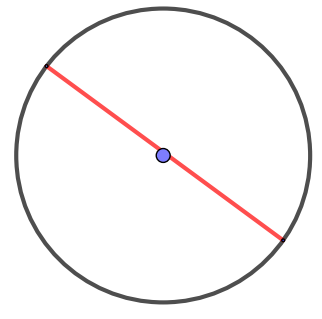

$n=1 \rightarrow 2$ regiões

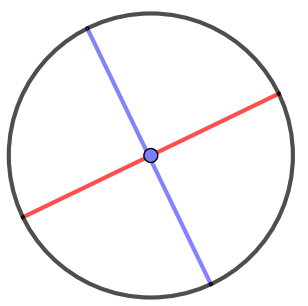

$n=2 \rightarrow 4$ regiões

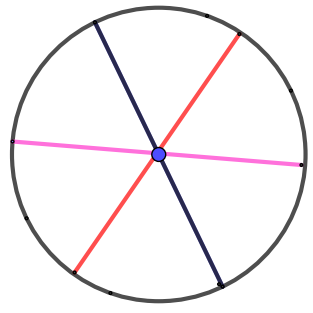

$n=3 \rightarrow 6$ regiões

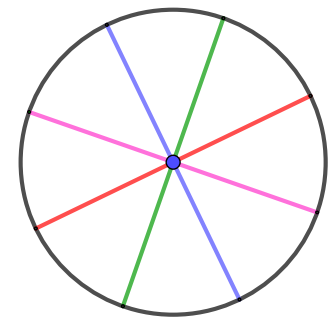

$n=4 \rightarrow 8$ regiões

Figura 3: Número de regiões de uma pizza com $n$ cortes retos concorrendo em um mesmo ponto

Cortes mais gerais também podem ser explorados como vários concorrentes num mesmo ponto e alguns não, ou mesmo com todos concorrentes dois a dois em pontos distintos.

\subsection{Cortes gerais de uma pizza}

Consideremos agora o caso mais geral com $n$ cortes lineares de uma pizza não tendo dois paralelos e nem três deles concorrentes num mesmo ponto para obtermos o máximo de regiões, conforme Figura 4, mostrando quatro cortes e 11 partes. 


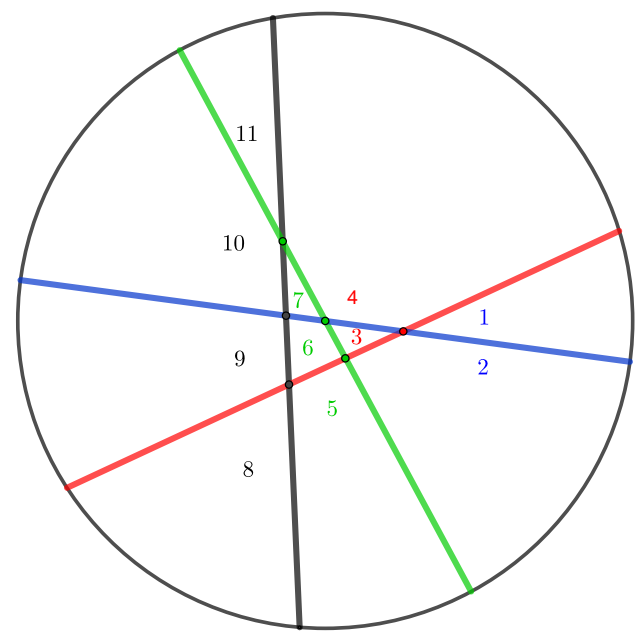

Figura 4: Quatro cortes retilíneos dividindo uma pizza em 11 pedaços

Observamos que o $n$-ésimo corte, não paralelo a nenhum dos $n-1$ cortes anteriores e não cruzando as interseções já existentes, cortará todos os anteriores em $n-1$ pontos distintos e acrescentará mais $n$ partes convexas, que podem ser contadas passo a passo de modo que podemos descobrir o padrão:

$$
\begin{aligned}
& p(0)=1, \\
& p(1)=p(0)+1=1+1=2, \\
& p(2)=p(1)+2=2+2=4, \\
& p(3)=p(2)+3=4+3=7, \\
& \vdots=\vdots \\
& p(n)=p(n-1)+n, n \in \mathbb{N}
\end{aligned}
$$

Para encontrar a função discreta $p(n)$ explicitada em função de $n, n \in \mathbb{N}$, solução da equação (2), propomos explorar vários conteúdos matemáticos como alguns que citaremos a seguir.

\subsection{Operando com as relações de $p(n), n \in \mathbb{N}$}

Somando membro a membro todas as relações anteriores dadas pela equação discreta (2), para $n=0,1,2 \ldots$ obtemos:

$p(0)+p(1)+\ldots+p(n-1)+p(n)=1+(p(0)+1)+(p(1)+2)+\ldots+(p(n-2)+n-1)+(p(n-1)+n)$ 
Simplificando a expressão anterior, o número máximo de regiões do plano cortadas por $n$ retas é:

$$
p(n)=1+\underbrace{(1+2+\cdots+n)}=1+\underbrace{\frac{n(n+1)}{2}}=\frac{n^{2}+n+2}{2}, n \in \mathbb{N} .
$$

Observamos que, cada $n$ cortes, o número de partes $p(n)=1+\frac{n(n+1)}{2}$ é igual a 1 mais um número triangular $T_{n}=\frac{n(n+1)}{2}$, o que nos dá margem para explorar os números figurados. Também $T_{n}=\frac{n(n+1)}{2}$ representa a soma dos $n$ primeiros naturais, o que pode ser mostrado de várias maneiras.

\subsection{Tabela e gráficos}

As primeiras tentativas para resolver o problema geralmente são de ilustração gráfica dos primeiros cortes e regiões levando à construção de gráficos, planilhas e a obtenção da sequência de partes da pizza $1,2,4,7,11, \ldots$ correspondentes a $n=0,1,2,3,4, \ldots$ cortes retilíneos obtidos da equação (2) com a condição inicial $p(0)=1$.

Neste caso, o número $n$ de cortes gerais forma uma progressão aritmética (PA) associada ao número $p_{3}(n)$ de regiões da pizza cortadas por eles, que é uma outra PA de segunda ordem, ou seja, uma sequência em que as diferenças primeiras $\Delta p_{3}(n)$ entre seus termos consecutivos formam uma PA, exatamente igual a $r(n)$ e, consequentemente, as diferenças segundas $\Delta^{(2)} p_{3}(n)$ são constantes, conforme mostra a Tabela 2.

\begin{tabular}{|c|c|c|c|c|c|c|c|c|c|}
\hline$n$ (cortes) & 0 & 1 & 2 & 3 & 4 & 5 & 6 & 7 & $\cdots$ \\
\hline$p_{1}(n)$ (retas paralelas distintas) & 1 & 2 & 3 & 4 & 5 & 6 & 7 & 8 & $\cdots$ \\
\hline$\Delta p_{1}(n)$ & 1 & 1 & 1 & 1 & 1 & 1 & 1 & 1 & $\cdots$ \\
\hline$p_{2}(n)(p i z z a:$ retas concorrentes num mesmo ponto) & 1 & 2 & 4 & 6 & 8 & 10 & 12 & 14 & $\cdots$ \\
\hline$\Delta p_{2}(n)$ (Diferença primeira) & 1 & 2 & 2 & 2 & 2 & 2 & 2 & 2 & $\cdots$ \\
\hline$p_{3}(n)$ (retas concorrentes em pontos distintos) & $\mathbf{1}$ & $\mathbf{2}$ & $\mathbf{4}$ & $\mathbf{7}$ & $\mathbf{1 1}$ & $\mathbf{1 6}$ & $\mathbf{2 2}$ & $\mathbf{2 9}$ & $\cdots$ \\
\hline$\Delta p_{3}(n)=r(n)$ (Diferenças primeira) & 1 & 2 & 3 & 4 & 5 & 6 & 7 & 8 & $\cdots$ \\
\hline$\Delta^{(2)} p_{3}(n)$ (Diferenças segunda) & 1 & 1 & 1 & 1 & 1 & 1 & 1 & 1 & $\cdots$ \\
\hline
\end{tabular}

Tabela 2: $n$ (cortes distintos dividindo a $p i z z a), p(n)$ (partes) e $\Delta p(n)$ (diferenças)

Vemos graficamente como as partes vão aumentando quadraticamente como mostra a Figura 5 a seguir. 


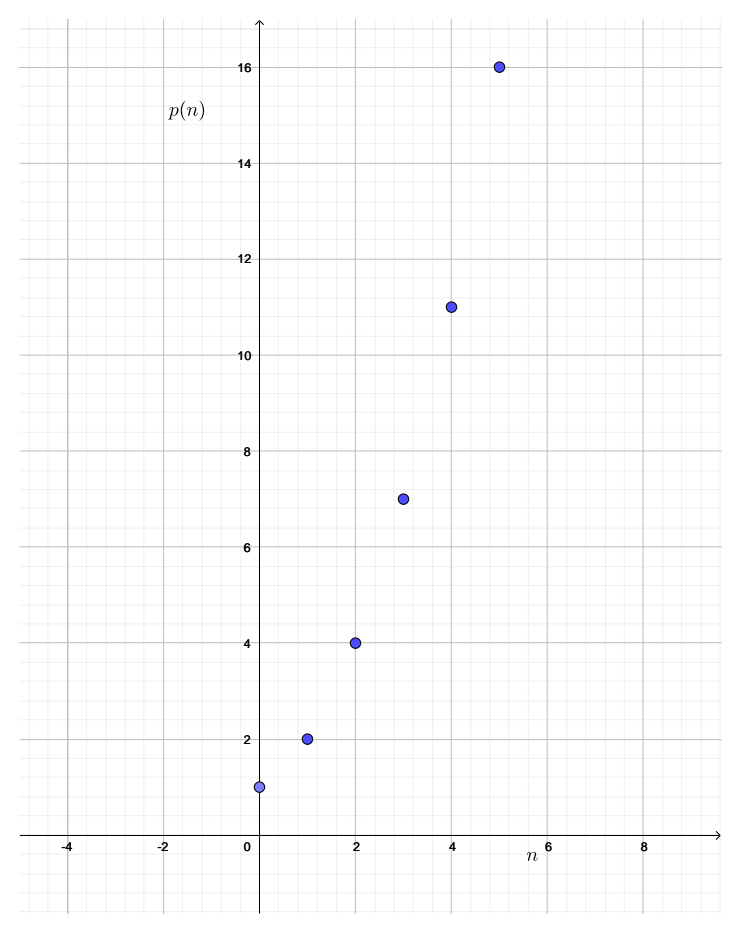

Figura 5: Máximo de partes $p(n)$ de uma pizza com $n$ cortes retilíneos

\subsection{Usando o princípio da indução matemática}

Para demonstrar que a proposição $p(n)=1+\frac{n(n+1)}{2}$ estabelecida para cada número natural $n$ é válida para todo $n \in \mathbb{N}$, podemos usar Indução Matemática, que é uma técnica de demonstração direta, adequadas proposições que poderão ser válidas para todos números naturais. A Indução Matemática sobre $n$ pode ser usada neste caso de acordo com os seguintes passos:

a) Verificamos inicialmente a Base da Indução (BI), o primeiro passo indutivo de que a proposição dada é válida para um primeiro número natural $n_{0}$. De fato, verificamos que para $n_{0}=0$ temos $p(0)=1$ (com zero corte temos a pizza inteira), o que é válido.

b) Admitamos em seguida a Hipótese de Indução (HI) de que $p(n)=1+\frac{n(n+1)}{2}$ é uma propriedade válida para um certo valor de $n \in \mathbb{N}, n>n_{0}$.

c) Para concluir que $p(n)$ é válida para todo número natural, verificamos se ela também vale para o próximo número natural $n+1$. Para isso, observamos que o $(n+1)$-ésimo corte deve atravessar todos os $n$ cortes anteriores por fora das regiões limitadas, produzindo no máximo outras $n+1$ regiões distintas, de modo que

$$
\underbrace{[1+(1+2+\cdots+n)]}+n+1 \stackrel{H I}{=}[\underbrace{\left[1+\frac{n(n+1)}{2}\right]}+n+1=
$$




$$
=1+\frac{n(n+1)}{2}+\frac{2(n+1)}{2}=1+\frac{(n+1)(n+2)}{2},
$$

que é exatamente a proposição para $n+1$. Assim, a proposição é válida para todo $n \in \mathbb{N}$.

\subsection{Usando o princípio da contagem}

Para obtermos a relação do número máximo de partes da pizza percebemos que, a partir do terceiro corte retilíneo, um novo corte retilíneo dividirá todas as partes em duas, com exceção das regiões existentes correspondentes à interseção de todas as outras duas a duas. Novas partes são obtidas duplicando o número de partes anteriores e subtraindo a quantidade de combinações duas a duas dos cortes anteriores que já existiam, ou seja:

$$
p(n)=2 p(n-1)-\left(\begin{array}{c}
n-1 \\
2
\end{array}\right)=2 p(n-1)-\frac{(n-1)(n-2)}{2}, \quad n=3,4,5, \ldots
$$

E também, conforme em Pitombeira (2009), podemos escrever o que equivale ao número máximo de regiões do plano cortado por $n$ retas usando combinação como

$$
p(n)=\left(\begin{array}{l}
n \\
0
\end{array}\right)+\left(\begin{array}{l}
n \\
1
\end{array}\right)+\left(\begin{array}{l}
n \\
2
\end{array}\right)=1+\left(\begin{array}{c}
n+1 \\
2
\end{array}\right)=1+n+\frac{n(n-1)}{2}, n \in \mathbb{N}
$$

Além disso, a solução do problema das partes da pizza com $n$ cortes, $n \in \mathbb{N}$ com contagem e indução pode ser vista considerando um arranjo $A$ de $n$ retas dividindo um plano em $p(A)$ regiões convexas, conforme [1].

Se as retas forem todas paralelas, temos $p(A)=n+1$ regiões ilimitadas (como no caso das quebras de um grissini ou dos $n$ pontos dividindo uma reta).

Suponhamos que nem todas retas sejam paralelas, de modo que o conjunto $C$ dos pontos de interseções de retas de $A$ contidos numa circunferência não é vazio. Seja um ponto de interseção $P \in C$ e $r_{P}$ a quantidade de retas de $A$ que se interceptam em $P$. Por Indução Matemática sobre $n \geq 3$ retas mostramos que:

$$
p(A)=1+n+\sum_{P \in C}\left(r_{P}-1\right)
$$

De fato; considerando a base de indução

(BI) Se tivermos só uma reta, $n=1$ então $C=\emptyset$, pois não há outra reta para interceptá-la, temos $p(A)=1+1=2$ partes.

Observemos que para $n=2$ retas temos $p(A)=1+2+(2-1)=4$, o que também condiz com a situação em que temos duas retas concorrentes num ponto $P$ do plano, dividindo-o em 4 regiões ilimitadas.

Adotamos agora a hipótese de indução

(HI) Supomos que a fórmula (4) $p(A)=1+n-1+\sum_{P \in C}\left(r_{P}-1\right)$ seja válida para $n-1, n \geq 3$ retas cortando o plano. 
Para verificarmos que a mesma é válida para todo n natural, consideremos um arranjo $A$ com $n \geq 3$ retas. Ao retirarmos desse arranjo uma de suas retas, ficamos com um arranjo $A^{\prime}$ com $n-1$ retas, em que vale (4).

Ao reintroduzirmos nesse arranjo mais uma reta $r_{L}$, e a deslizamos de um lado para o outro do plano, de modo que ela não cruze nenhuma das regiões limitadas já existentes, ela encontrará as $k$ retas existentes criando $k+1$ novas regiões.

Chamando de $C_{L}$ o conjunto dos $k$ pontos em que $r_{L}$ encontra as outras $k$ retas.temos:

$$
p\left(A^{\prime}\right)=1+(n-1)+\sum_{P \in C-C_{L}}\left(r_{P}-1\right)+\sum_{P \in C_{L}}\left(r_{p}-2\right)
$$

$\log$,

$$
\begin{gathered}
p(A)=p\left(A^{\prime}\right)+n=1+(n-1)+\sum_{P \in C-C_{L}}\left(r_{P}-1\right)+\sum_{P \in C_{L}}\left(r_{P}-2\right)+k+1 \\
=1+(n-1)+\sum_{P \in C-C_{L}}\left(r_{P}-1\right)+\sum_{P \in C_{L}}\left(r_{P}-1\right)-k+k+1= \\
=1+n+\sum_{P \in C}\left(r_{P}-1\right),
\end{gathered}
$$

e, assim, a fórmula (4) é válida para todo $n \in \mathbb{N}$.

Observamos, ainda, que a quantidade de regiões ilimitadas num arranjo de $n$ retas gerais é $2 n$, pois cada reta divide a região do plano em duas partes. Para ver isso, consideramos uma grande circunferência que contenha todos os pontos de cruzamento das retas. As retas não se interceptam fora da circunferência e como cada uma delas intercepta a circunferência em $2 n$ pontos conforme Figuras 3 e 4, seus prolongamentos produzem a quantidade de regiões ilimitadas que são iguais a $2 n$.

Sendo $A$ um arranjo de $n$ retas em um plano em posição geral. Indicaremos por $p(n)$ a quantidade de regiões determinadas pelas $n$ retas. Notemos que $p(1)=2, p(2)=2+2=4, p(3)=2+2+3=7$, e assim por diante. Sempre que aumentamos uma reta, acrescentamos tantas regiões quantas são as retas, de modo que:

$$
p(n)=2+2+3+4+\cdots+n=1+(1+2+3+\cdots+n)=1+\frac{n(n+1)}{2}=\frac{1}{2}\left(n^{2}+n+2\right)
$$

Podemos também provar por indução matemática seguindo o mesmo procedimento anterior. Ao reintroduzirmos a reta $r_{L}$ num arranjo com $n$ retas, criamos $n+1$ novas regiões, de modo que

$$
p(n+1)=p(n)+n+1=\frac{1}{2}\left(n^{2}+n+2\right)+n+1=\frac{1}{2}\left[(n+1)^{2}+(n+1)+2\right], \quad n \in \mathbb{N} .
$$

Observação: Se um arranjo $A$ tem $n$ retas em posição geral, o conjunto $C$

$$
\left(\begin{array}{l}
n \\
2
\end{array}\right)=\frac{n !}{2 !(n-2) !}=\frac{n(n-1)}{2}
$$

pontos, e para cada $P \in C$ temos $r_{P}=2$. Logo, a fórmula da solução (4) realmente é:

$$
p(A)=1+n+\sum_{P \in C}\left(r_{P}-1\right)=1+n+\sum_{P \in C} 1=1+n+\frac{n(n-1)}{2}=\frac{1}{2}\left(n^{2}+n+2\right) .
$$




\subsection{Usando grafos}

A teoria dos grafos é bastante usada no estudo das relações entre objetos de um determinado conjunto. Grafos são estruturas do tipo $G(V, A)$, em que $V$ é um conjunto não vazio de objetos denominados vértices e $A$ arestas, subconjuntos de pares não ordenados de $V$.

O número máximo de regiões do plano dividido por $n$ retas acontece quando elas estão em posição geral e a configuração em que possuem o número máximo de pontos de intersecção entre elas, assim, podemos pensar num grafo plano $G$ para determinar o número $p(n)$ de regiões em que elas dividem o plano. De fato, seja um círculo grande contendo em seu interior todos os pontos de interseção das $n$ retas. Para cada duas das $n$ retas dadas, há um ponto de intersecção e vice-versa. Logo, o número dos pontos de intersecção, todos situados no interior do nosso círculo, é $\frac{n(n-1)}{2}$ mais os $2 n$ pontos em que as $n$ retas interceptam a circunferência, considerados como vértices do grafo $G$.

Temos, assim, o número de vértices do grafo $G$ dado por:

$$
V=\frac{n(n-1)}{2}+2 n
$$

As arestas de $G$ podem ser identificadas como os $2 n$ arcos de circunferência com extremidades nos pontos onde as $n$ retas a cortam, mais os segmentos de reta interiores, que ligam os pontos de interseção de retas.

Cada uma das $n$ retas possuem $n+1$ vértices, sendo $n-1$ deles correspondente a intersecção de cada uma com as outras $n-1$ retas mais os 2 pontos em que ela corta a circunferência. Assim, $n$ segmentos formam $n$ arestas do grafo $G$ sobre cada uma das $n$ retas dadas, de modo que as $n^{2}$ arestas de $G$ são interiores e as $2 n$ arestas que estão sobre a circunferência, totalizando $A=n^{2}+2 n$ arestas.

Considerando a fórmula de Euler $V-A+F=2$ conforme [3], para um grafo com $V$ vértices e $A$ arestas e decompondo o plano em $F=p(n)$ regiões, o número máximo $p(n)$ de regiões em que as $n$ retas dividem o plano é igual ao número de regiões determinadas pelo grafo $G$ menos a região exterior ao círculo:

$$
V-A+p(n)=1 \Longleftrightarrow\left(\frac{n(n-1)}{2}+2 n\right)-\left(n^{2}+2 n\right)+p(n)=1 \Longleftrightarrow p(n)=1+\frac{n(n+1)}{2} .
$$

o que concorda com a solução (3) da equação (2).

\subsection{Usando sistema linear}

Como os $n$ cortes retilíneos dividem a pizza bidimensional em $p(n), n \geq 1$ pedaços planos, podemos supor que a função discreta $p(n)$ representando o número máximo de pedaços seja uma função polinomial de segunda ordem, $p(n)=b_{2} n^{2}+b_{1} n+b_{0}$ em que os coeficientes $b_{0}, b_{1}$ e $b_{2}$ devem ser determinados.

De fato, usando os primeiros termos de $p(n)$, para $n=0,1,2$, obtemos o sistema de equações 
lineares de Vandermonde

$$
\left\{\begin{array}{c}
p(0)=1=b_{2} 0^{2}+b_{1} 0+b_{0} \\
p(1)=2=b_{2} 1^{2}+b_{1} 1+b_{0} \\
p(2)=4=b_{2} 2^{2}+b_{1} 2+b_{0}
\end{array}\right.
$$

que pode ser explorado matricialmente

$$
\left[\begin{array}{ccc}
1 & 0 & 0 \\
1 & 1 & 1 \\
1 & 2 & 2^{2}
\end{array}\right]\left[\begin{array}{l}
b_{0} \\
b_{1} \\
b_{2}
\end{array}\right]=\left[\begin{array}{l}
1 \\
2 \\
4
\end{array}\right]
$$

e, cuja solução, pode ser obtida introduzindo ou revisando os métodos de resolução de sistemas lineares, que é: $b_{0}=1, b_{1}=\frac{1}{2}$ e $b_{2}=\frac{1}{2}$. Assim, segue o resultado desejado:

$$
p(n)=\frac{1}{2} n^{2}+\frac{1}{2} n+1=\frac{n^{2}+n+2}{2}=1+\frac{n(n+1)}{2} .
$$

\subsection{Equação discreta com uma condição inicial}

A equação discreta linear não homogênea de primeira ordem $p(n)=p(n-1)+n, n \geq 1$ com a condição inicial $p(0)=1$ pode ser resolvida como a soma de uma solução da equação homogênea associada mais uma solução particular da equação não homogênea:

a) Encontramos uma solução $p_{h}(n)$ da equação discreta homogênea associada $p(n)=p(n-1)$, fazendo $p_{h}(n)=\lambda^{n}, \lambda \neq 0, \lambda \in \mathbb{R}$. Temos, então, $\lambda^{n}=\lambda^{n-1} \Longleftrightarrow \lambda^{n-1}(\lambda-1)=0 \Longrightarrow \lambda=1$.

Assim qualquer combinação de $\lambda=1$ será uma solução da equação homogênea, o que nos dará uma constante como solução geral, $p_{h}(n)=a_{0} * 1, a_{0} \in \mathbb{N}$.

b) Encontrando uma solução particular da equação não homogênea do tipo:

$$
p_{n h}(n)=\left(a_{2} n+a_{1}\right) n
$$

com $a_{i}, i=1,2$ constantes a serem determinadas, já que uma constante é solução da equação homogênea e a parte não homogênea é um polinômio de grau em $n$. Substituindo $p_{n h}(n)$ na equação geral, obtemos: $\left(a_{2} n+a_{1}\right) n=\left(a_{2}(n-1)+a_{1}\right)(n-1)+n$ que nos dá $a_{2}=\frac{1}{2}=a_{1}$. Portanto $p(n)=\frac{1}{2} n^{2}+\frac{1}{2} n+a_{0}, n \in \mathbb{N}$, e com a condição de que $p(0)=1$, concluímos que $a_{0}=1$ e a solução desejada fica $p(n)=\frac{1}{2} n^{2}+\frac{1}{2} n+1, n \in \mathbb{N}$.

Assim, ao abordarmos o problema do corte da pizza podemos aprofundar os estudos de cada conteúdo envolvido, desde definições, e a formalização dos resultados relacionados.

\section{Cortando bolo com $n$ cortes}

Após explorar os problemas mais simples, abordamos o problema de encontrar quantas partes $E(n)$ obtemos de um bolo com $n$ cortes planos.

Para $n=0$ corte, temos uma parte inteira $E(0)=1$. 
Um corte retilíneo contínuo, para $n=1$ temos o bolo dividido em duas partes $E(1)=2$.

Com dois ou mais cortes planos no bolo, o problema já não apresenta uma resposta única, pois depende da posição relativa dos cortes planos, ou seja, como eles se dispõem: paralelos, concorrentes e como eles se cruzam entre si, como no caso do espaço cortado por $n$ planos.

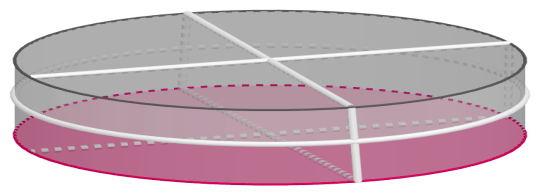

Figura 6: Dois cortes planos verticais e um corte horizontal dividindo um bolo

Se fizermos dois ou três cortes planos paralelos teremos $E(2)=3, E(3)=4, \ldots$ partes, que é um caso análogo aos das quebras do grissini ou dos cortes paralelos de uma pizza plana.

Se os cortes planos forem concorrentes tendo uma reta em comum, teremos $E(2)=4$ partes ou regiões, que é o número máximo de regiões que divide o bolo neste caso.

Se dois cortes planos forem paralelos e o outro concorrente com os dois anteriores, teremos o bolo dividido em 6 regiões.

Às vezes aparece em desafios e diversões matemáticas sobre a pergunta qual é o maior número de pedaços que podemos obter ao dividir um bolo com 3 cortes apenas.

É fácil ver que dois cortes verticais (ortogonais) passando pelo centro corta o bolo em 4 partes, e um corte ortogonal a eles passando horizontalmente pelo meio do bolo, leva-nos a obter 8 pedaços conforme Figura 6. Analogamente aos três planos concorrentes num mesmo ponto, teremos 8 regiões, como acontece com os planos cartesianos triortogonais que dividem o espaço tridimensional em 8 octantes.

Observemos que os primeiros termos do número máximo de regiões $E(n)$ podem ser descobertos por tentativas de visualização, mas obter a função $E(n)$ para um número grande $n$ de cortes planos no bolo fica cada vez mais difícil.

Geralmente começamos visualizando as configurações, calculando o número de regiões em que o bolo é dividido por $n$ cortes planos distintos e tabelando os pares $(n, E(n))$ para alguns valores iniciais de $n$, como $n=0,1,2,3$. Para o caso geral, consideramos um número $n$ de cortes planos do bolo cuja interseção, três a três, seja unicamente um ponto distinto de todas as outras interseções para obtermos o número máximo de pedaços. 


\subsection{Descobrindo um padrão para as regiões}

O número máximo de partes do bolo obtido com $n$ cortes planos, no caso geral, pode ser obtido segundo o padrão:

$$
\begin{aligned}
E(0) & =1, \\
E(1) & =E(0)+1=1+1=2, \\
E(2) & =E(1)+1+1=4, \\
E(3) & =E(2)+1+3=8, \\
\quad & =\vdots \\
E(n) & =E(n-1)+1+\frac{(n-1) n}{2}, n \in \mathbb{N}
\end{aligned}
$$

Assim, o número máximo de partes obtidas com $n+1$ cortes planos gerais é dado por (6), ou seja, é igual ao número de regiões cortadas por $n$ planos mais 1 e mais o número triangular de ordem $n, \frac{(n-1) n)}{2}$.

A função discreta $E(n)$ pode ser explorada com vários conteúdos matemáticos, como no caso dos cortes retilíneos da pizza ou das quebras do grissini, como descreveremos a seguir.

\subsection{Uso de Tabelas e Gráficos}

Sendo $E_{p}(n)$ o número de pedaços obtidos com $n$ cortes planos paralelos no bolo, $E_{c m r}$ como com $n$ cortes planos concorrentes passando numa mesma reta, e $E(n)$ o número de partes obtidas com $n$ cortes planos gerais, não paralelos nem passando pela mesma reta e nem mais do que três deles se interceptando num mesmo ponto. Podemos construir os primeiros termos das sequências como na Tabela 3 desenhando e tentando visualizar, ou usando o padrão (6).

\begin{tabular}{|c|c|c|c|c|c|c|c|c|c|c|c|}
\hline$n$ (planos) & 0 & 1 & 2 & 3 & 4 & 5 & 6 & 7 & 8 & 9 & $\cdots$ \\
\hline$E_{p}(n)$ (planos paralelos) & 1 & 2 & 3 & 4 & 5 & 6 & 7 & 8 & 9 & 10 & $\cdots$ \\
\hline$\Delta E_{p}(n)$ & 1 & 1 & 1 & 1 & 1 & 1 & 1 & 1 & 1 & 1 & $\cdots$ \\
\hline $\begin{array}{c}E_{c m r}(n) \text { (planos concorrentes } \\
\text { na mesma reta) }\end{array}$ & 1 & 2 & 4 & 6 & 8 & 10 & 12 & 14 & 16 & 18 & $\cdots$ \\
\hline$\Delta E_{c m r}(n)$ & 1 & 2 & 2 & 2 & 2 & 2 & 2 & 2 & 2 & 2 & $\cdots$ \\
\hline$E(n)$ (planos gerais) & $\mathbf{1}$ & $\mathbf{2}$ & $\mathbf{4}$ & $\mathbf{8}$ & $\mathbf{1 5}$ & $\mathbf{2 6}$ & $\mathbf{4 2}$ & $\mathbf{6 4}$ & $\mathbf{9 3}$ & $\mathbf{1 3 0}$ & $\cdots$ \\
\hline$\Delta E(n)=p(n)$ & 1 & 2 & 4 & 7 & 11 & 16 & 22 & 29 & 37 & 46 & $\cdots$ \\
\hline$\Delta_{2} E(n)=r(n)$ & 1 & 2 & 3 & 4 & 5 & 6 & 7 & 8 & 9 & 10 & $\cdots$ \\
\hline$\Delta_{3} E(n)$ & 1 & 1 & 1 & 1 & 1 & 1 & 1 & 1 & 1 & 1 & $\cdots$ \\
\hline
\end{tabular}

Tabela 3: $n$ (cortes planos distintos dividindo o bolo $) \times E(n)$ (partes ou pedaços obtidos)

Observamos na Tabela 3 que a diferença $\Delta E(n)$ é exatamente igual a $p(n)$ o número máximo de regiões do plano cortado por $n$ retas, $\Delta_{2} E(n)$ é uma progressão aritmética de razão igual a $1 \mathrm{e}$ $\Delta_{3} E(n)$ é constante. 


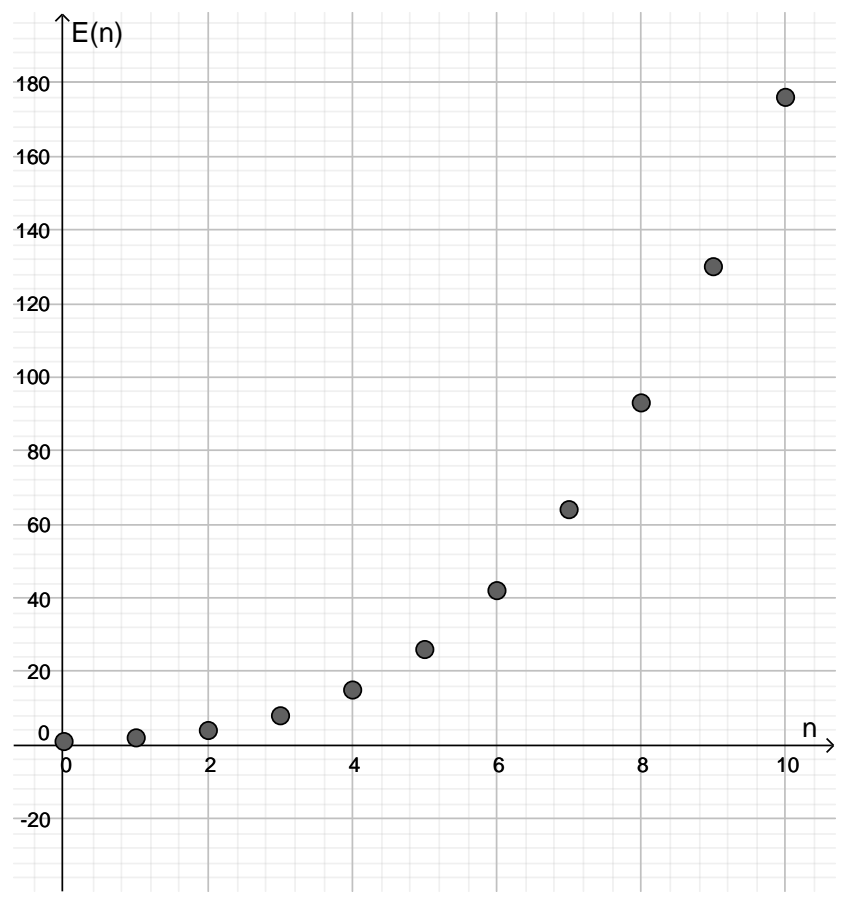

Figura 7: Número de regiões do espaço cortadas por $n$ planos

E a representação gráfica da função discreta $E(n)=E(n-1)+1+\frac{(n-1) n}{2}=\frac{1}{6} n^{3}+\frac{5}{6} n+1$ pode ser vista para alguns valores iniciais de $n$ na Figura 7.

\subsection{Usando Sistema Linear}

Suponhamos que a relação entre o número $n$ de cortes planos de um bolo e o respectivo número máximo de partes $E(n)$ obtidas seja uma função polinomial do terceiro grau do tipo:

$$
E(n)=c_{3} n^{3}+c_{2} n^{2}+c_{1} n+c_{0}
$$

Utilizando os valores iniciais, fáceis de serem obtidos, exploramos o sistema linear de Vandermonde correspondente:

$$
\left\{\begin{array}{l}
E(0)=c_{0}=1 \\
E(1)=c_{3} 1^{3}+c_{2} 1^{2}+c_{1} 1+c_{0}=2 \\
E(2)=c_{3} 2^{3}+c_{2} 2^{2}+c_{1} 2+c_{0}=4 \\
E(3)=c_{3} 3^{3}+c_{2} 3^{2}+c_{1} 3+c_{0}=8
\end{array}\right.
$$

Resolvendo o sistema linear (7) obtemos os valores dos coeficientes: $c_{0}=1, c_{1}=\frac{5}{6}, c_{2}=0, c_{3}=\frac{1}{6}$ e, portanto, a função discreta solução:

$$
E(n)=\frac{1}{6} n^{3}+\frac{1}{6} 5 n+\frac{6}{6} \approx 0,17 n^{3}+0,83 n+1, n \in \mathbb{N} .
$$


que gera a sequência solução dos números de pedaços possíveis: 1, 2, 4, 8, 15, 26, 42, 64, 93, 130, 176,232

\subsection{Usando Combinação}

Outro modo de obter uma relação equivalente para o número de partes do bolo é considerar que, a partir do quarto corte plano, um novo plano dividirá todas as partes em duas, com exceção das regiões correspondentes à interseção de todas as outras partes combinadas três a três, análogo ao que ocorreu no problema dos cortes retilíneos da pizza plana.

Para isso, duplicamos o número de partes anteriores e subtraímos a quantidade de combinações três a três dos cortes planos que já existiam, ou seja:

$$
E(n)=2 E(n-1)-\left(\begin{array}{c}
n-1 \\
3
\end{array}\right)=2 E(n-1)-\frac{(n-1)(n-2)(n-3)}{6}=\left(\begin{array}{c}
n+1 \\
3
\end{array}\right)+n+1
$$

que nos dá a sequência de pedaços conforme vamos aumentando os cortes planos: $1,2,4,8,15,26,42, \ldots$ Usando a relação binomial de Stifel [3], o número máximo $E(n)$ de pedaços de um bolo obtidos por $n$ cortes planos também pode ser escrito como

$$
E(n)=\left(\begin{array}{l}
n \\
0
\end{array}\right)+\left(\begin{array}{l}
n \\
1
\end{array}\right)+\left(\begin{array}{l}
n \\
2
\end{array}\right)+\left(\begin{array}{l}
n \\
3
\end{array}\right)
$$

de modo análogo ao mostrado por [5].

\subsection{Equação discreta com uma condição inicial}

A equação discreta linear não homogênea de primeira ordem com coeficientes constantes

$$
E(n)=E(n-1)+1+\frac{(n-1) n}{2}, n \in \mathbb{N}
$$

com a condição inicial $E(0)=1$ pode ser resolvida encontrando uma solução da equação discreta homogênea associada $E(n)=E(n-1)$ que será uma constante $a_{0}$, mais uma solução particular da equação não homogênea (7), que supomos do tipo:

$$
E_{p}(n)=\left(a_{3} n^{2}+a_{2} n+a_{1}\right) n
$$

com $a_{i}, i=1,2,3$ constantes a serem determinadas. Substituindo na equação geral essa solução particular, obtemos

$$
a_{3} n^{3}+a_{2} n^{2}+a_{1} n=a_{3}(n-1)^{3}+a_{2}(n-1)^{2}+a_{1}(n-1)+1+\frac{(n-1) n}{2}
$$

E, simplificando, fica

$$
a_{3}\left(-3 n^{2}+3 n-1\right)+a_{2}(-2 n+1)+a_{1}+1+\frac{1}{2} n^{2}-\frac{1}{2} n=0
$$


Colocando em evidência os coeficientes das parcelas polinomiais de mesmo grau de $n$, obtemos um polinômio nulo:

$$
\left(-3 a_{3}+\frac{1}{2}\right) n^{2}+\left(3 a_{3}-2 a_{2}-\frac{1}{2}\right) n+\left(-3 a_{3}+a_{2}+a_{1}+1\right)=0 .
$$

Portanto, igualando os seus coeficientes a zero, obtemos os valores das constantes $a_{3}=\frac{1}{6}, a_{2}=0$ e $a_{1}=\frac{5}{6}$, e portanto a solução geral será do tipo:

$$
E(n)=\frac{1}{6} n^{3}+\frac{5}{6} n+a_{0}
$$

que usando a condição inicial $E(0)=1$ dá-nos $a_{0}=1$ e a solução encontrada é a desejada

$$
E(n)=\frac{1}{6} n^{3}+\frac{5}{6} n+1 \text {. }
$$

\section{Relação do problema numa dimensão com o de outra menor}

É interessante observarmos que podemos escrever a relação de recorrência para o número máximo de pedaços $p(n-1)$ de uma pizza bidimensional (2D) cortada retilineamente $n-1$ vezes com o do número máximo de partes de um grissini unidimensional (1D) $r(n-1)$ quebrado $n-1$ vezes para obtermos o número máximo de pedaços $p(n)$ da pizza. Da mesma forma podemos obter as partes $E(n)$ de um bolo tridimensional (3D) cortados por $n$ planos com as partes cortadas por $n-1$ cortes planos anteriores mais o número de partes da pizza (2D) cortada retilineamente por $n-1$ cortes.

\subsection{Relacionando as partes da pizza (2D) com as partes do grissini (1D)}

Observamos inicialmente que podemos relacionar o número máximo de regiões (bidimensional) $p(n)$ de uma pizza com as partes unidimensionais de grissinis escrevendo o número máximo de pedaços de pizza como:

$p(n)=p(n-1)+n, \quad \Longleftrightarrow \quad p(n)=p(n-1)+r(n-1), n \in \mathbb{N}, \quad$ com $\quad p(0)=1 \quad$ e $\quad r(0)=1$.

em que $r(n-1)$ é o número máximo de regiões que $n-1$ quebras faz num grissini, como visto na seção 3 .

\subsection{Relacionando as partes do bolo (3D) com as da pizza (2D)}

Os primeiros valores das partes $E(n)$ de um bolo podem ser descobertos imaginando o $n$ - ésimo corte retilíneo deslizando no bolo sobre os $n-1$ cortes retilíneos anteriores e relacionando com o número $p(n-1)$ de $n-1$ cortes retilíneos de uma pizza conforme seção 3 . De fato,

$$
E(n)=E(n-1)+1+\frac{(n-1) n}{2}
$$

pode ser reescrita como:

$$
E(n)=E(n-1)+p(n-1) \quad \text { com } \quad E(0)=1 \quad \text { e } \quad p(0)=1 .
$$


Que também nos dá a sequência do número máximo de regiões do bolo com $n$ cortes planos é $1,1+1=2,2+2=4,4+4=8,8+7=15,15+11=26, \ldots$ e,

$$
E(n)=\frac{(n-1)^{3}+5(n-1)+6}{6}+\frac{(n-1)^{2}+(n-1)+2}{2}=\frac{1}{6} n^{3}+\frac{1}{6} 5 n+\frac{6}{6}, n \in \mathbb{N} .
$$

Conhecidas as fórmulas de recorrência para o número máximo de pedaços ao cortar linearmente uma pizza plana e o bolo tridimensional em $n$ partes, podemos questionar e lançar um problema mais geral sobre qual é o maior número de partes que $n$ objetos $(k-1)$ - dimensionais, $k \in \mathbb{N}$, corta um objeto de uma dimensão superior $k$ - dimensional no sentido de generalizar a equação (8) e incentivá-los a continuar explorando.

\section{Conclusão}

No processo de resolução de problemas em grupos, a maioria dos estudantes costuma apresentar dificuldades iniciais como no problema dos cortes de um bolo. Algumas vezes é necessário orientá-los na procura de um modo mais fácil para resolver o problema de modo a começar com um problema relacionado mais simples conforme sugere Verificamos que o problema de cortes de um grissini e de uma pizza foram mais fáceis de serem abordados e entendidos, dado que as primeiras ideias que surgem para a maioria dos grupos de estudantes são as tentativas de visualização geométrica.

Considerar as suposições e tentativas iniciais de visualização geométrica, de relacionar cada passo com o anterior para a descoberta de padrões e a investigação usando diferentes conteúdos matemáticos contribui para o resgate dos conhecimentos prévios, o desenvolvimento do raciocínio lógico e ampliar os horizontes de conhecimento defendidos por [4] e outros pesquisadores da resolução de problemas no ensino de matemática.

$\mathrm{Na}$ arte de resolver o problema dos cortes planos de um bolo, percebemos a riqueza da exploração de vários conteúdos matemáticos que a princípio poderiam ser apresentados ou revistos isoladamente, o que torna o processo de exploração matemática interessante, vislumbrando a abordagem da resolução de problemas no ensino, de forma integradora relacionada a vida social e familiar recomendado nos parâmetros curriculares da BNCC (Brasil, 2018) [2], nos projetos pedagógicos dos cursos de graduação em Matemática.

\section{Referências}

[1] Bjorner, A. e Stanley, R. P.,A Combinatorial Miscellany, Disponível in http://wwwmath.mit.edu/ rstan/papers/comb.pdf

[2] Brasil, Base Nacional Comum Curricular, Ensino Médio, Brasília, MEC/Secretaria da Educação Básica (2018).

[3] Lima, E. L., Carvalho, P. C. P., Morgado, A. C. e Wagner, E., A Matemática do Ensino Médio, Vol 2., $6^{a}$ Edição, Rio de Janeiro, SBM, (1999)

[4] Onuchic, L. R.; Allevato, N. S. G. Novas reflexões sobre o ensino-aprendizagem através da Resolução de Problemas. In: BICUDO, M. A. V.; BORBA, M. C. (Orgs.). Educação matemática: pesquisa em movimento. São Paulo: Cortez, 2005. 
[5] Pitombeira, J. B., RPM - Revista do Professor de Matemática. Disponível em http://rpm.org.br/cdrpm/10/10.htm acesso em: 05 de abril de 2019

[6] Polya, G., A arte de resolver problemas: um novo aspecto do método matemático. (How to solve it: a new aspect of mathematica method). Heitor Lisboa de Araújo (Trad.). Rio de Janeiro: Interciência, 2006.

[7] Salvador, J. A., Cortar bolo, pizza e palitos de pão explorando conteúdos matemáticos, IV Serp e I Sirp, 2017. Disponível em http://igce.rc.unesp.br/Home/Departamentos47/educacaomatematica/gterp/resumos-publicados-iv-serp-e-i-sirp.pdf acesso em: 5 de abril de 2019.

José Antonio Salvador

DM - CCET - UFSCar

<salvador@dm.ufscar.br>

Recebido: 15/11/2019

Publicado: 22/01/2020 